Moroccan J. of Pure and Appl. Anal. (MJPAA)

Volume 5(1), 2019, Pages 86-103

ISSN: Online 2351-8227 - Print 2605-6364

DOI 10.2478/mjpaa-2019-0007

\title{
Periodic solutions for a class of Duffing differential equations
}

\author{
Amina Feddaoui ${ }^{1, a}$, Jaume Llibre $^{2, b}$ and Amar MaKhlouf ${ }^{1, c}$
}

Авstract. We provide sufficient conditions for the existence of periodic solutions for the class of Duffing differential equations

$$
x^{\prime \prime}+c(t) x^{\prime}+a(t) x+b(t) x^{3}=h(t, x),
$$

where the functions $a(t), b(t), c(t)$ and $h(t, x)$ are $C^{2}$ and $T$-periodic in the variable $t$.

2010 Mathematics Subject Classification.34C15, 34C25, 37G15, 37C80, 37C30.

Key words and phrases. Periodic solution; averaging method; Duffing differential equation.

\section{Introduction and statement of the main results}

Several classes of Duffing differential equations have been investigated by many authors. They are mainly interested in the existence of periodic solutions, in their multiplicity, stability, bifurcations, ... see the survey of Mawhin [12], and also the articles [4]-[8], [13, 14, 17, 19] without trying to be exhaustive in the references.

In this work we study the periodic solutions for the class of Duffing differential equations

$$
x^{\prime \prime}+c(t) x^{\prime}+a(t) x+b(t) x^{3}=h(t, x)
$$

where the functions $a(t), b(t), c(t)$ and $h(t, x)$ are $C^{2}$ and $T$-periodic with respect to the variable $t$. As usual here the prime denotes derivative with respect to the time $t$.

In [18] the authors used the Leray-Schauder method to prove the existence of periodic solutions of the Duffing equation

$$
x^{\prime \prime}+c x^{\prime}+g(t, x)=h(t),
$$

where $c>0$ is fixed, $h$ and $g$ are $T$-periodic functions in $t$.

Received August 31, 2019 - Accepted October 06, 2019.

(C) The Author(s) 2017. This article is published with open access by Sidi Mohamed Ben Abdallah University.

${ }^{1}$ Department of Mathematics, University of Annaba, Laboratory LMA, P.O.Box 12, Annaba 23000, Algeria

2 Departament de Matematiques, Universitat Autònoma de Barcelona, 08193 Bellaterra, Barcelona, Catalonia, Spain

a e-mail: feddaouiamina90@gmail.com

${ }^{b}$ e-mail:makhloufamar@yahoo.fr

c e-mail: jllibre@mat.uab.cat. 
During the past three decades the existence and stability of periodic solutions for the equation (1.2), or for more general types of nonlinear second-order differential equations have been studied by many authors, see for instance $[5,8,13,17]$. Ortega initiated the study of the stability of periodic solutions of $(1.2)$ with $h(t)=0$ using the relation between topological degree and stability [14]. Besides the topological degree method, the method of upper and lower solutions is also successfully used to investigate stability properties. The techniques of these methods are different from the one of our method.

In [1] Benterki and Llibre studied the periodic solutions of equation (1.1) with $c(t)=c, h(t, x)=h(t), c>0$ is a constant, and $a(t), b(t)$ and $h(t)$ are continuous $T$-periodic functions. These differential equations were also studied by Chen and Li in the papers $[4,6]$. These authors studied the periodic solutions of the equation

$$
x^{\prime \prime}+c x^{\prime}+a(t) x+b(t) x^{3}=h(t),
$$

with the following additional conditions, either $b(t)>0, h(t)>0$ and $a(t)$ satisfies

$$
a(t) \leq \frac{\pi^{2}}{T^{2}}+\frac{c^{2}}{4}, \text { and } a_{0}=\frac{1}{T} \int_{0}^{T} a(t) d t>0,
$$

or $a(t)=a>0, b(t)=1$ and $c>0, a$ and $c$ are constants.

In [10] the authors studied the existence and the stability of periodic solutions of the Duffing differential equation (1.3) with $c=\varepsilon C>0, a(t)=\varepsilon^{2} A(t), A_{0} b_{0}>0$ where $A_{0}=\frac{1}{T} \int_{0}^{T} A(t) d t, b_{0}=\frac{1}{T} \int_{0}^{T} b(t) d t$ and $\varepsilon$ is sufficiently small.

In [2] Benterki and Llibre studied the existence of periodic solutions of the two Duffing differential equations $y^{\prime \prime}+a \sin y=b \sin t$ and $y^{\prime \prime}+a y-c y^{3}=b \sin t$, where $a, b$ and $c$ are real parameters.

In [9] Llibre and Makhlouf studied the periodic solutions of the second-order differential equation with variable potentials of form (1.1) with $c(t)=r(t)+\frac{p^{\prime}(t)}{p(t)}, a(t)=-\frac{q(t)}{p(t)}, b(t)=0$ and $h(t, x)=-\frac{f(t, x)}{p(t)}$, where the functions $p(t)>0, q(t), r(t)$ and $f(t, x)$ are $C^{2}$ and $T$-periodic in the variable $t$.

Instead of working with the T-periodic Duffing differential equation (1.1) we shall work with the following equivalent $T$-periodic differential system of first-order

$$
\begin{aligned}
& x^{\prime}=y, \\
& y^{\prime}=-c(t) y-a(t) x-b(t) x^{3}+h(t, x) .
\end{aligned}
$$

We define the functions

$$
\begin{array}{ll}
k(t)=\int_{0}^{t} c(s) d s, & m(t)=\int_{0}^{t} a(s) e^{k(s)} d s, \\
n(t)=\int_{0}^{t} b(s) e^{k(s)} d s, & g(t, x)=\int_{0}^{t} h(s, x) e^{k(s)} d s .
\end{array}
$$

Our results on the periodic solutions of the differential system (1.4) are summarized in the next two theorems. In these two theorems we provide 16 polynomial systems of the form $f_{1}\left(x_{0}, y_{0}\right)=f_{2}\left(x_{0}, y_{0}\right)=0$, the ones of Appendices 2 and 3, such that, under the assumptions of Theorems 1.1 or 1.2, for every simple zero $\left(x_{0}^{*}, y_{0}^{*}\right)$ of one of such polynomial systems the differential system (1.4) has a T-periodic solution $(x(t), y(t))$ such that $(x(0), y(0))$ is close to $\left(x_{0}^{*}, y_{0}^{*}\right)$.

Theorem 1.1. We consider the differential system (1.4) where the functions $a(t), b(t), c(t)$ and $h(t, x)$ are $C^{2}$ and $T-$ periodic in the variable $t$. Assume that the functions of (1.5) are T-periodic in the variable $t$. Then for every simple zero $\left(x_{0}^{*}, y_{0}^{*}\right)$ of one of polynomial systems (3.6)-(3.13) in the variables $x_{0}$ and $y_{0}$ given in Appendix 2, the differential system (1.4) has a T-periodic solution $(x(t), y(t))$ such that $(x(0), y(0))$ is close to $\left(x_{0}^{*}, y_{0}^{*}\right)$. 
Now we define the functions

$$
m(t)=\int_{0}^{t} a(s) d s, \quad n(t)=\int_{0}^{t} b(s) d s, \quad g(t, x)=\int_{0}^{t} h(s, x) d s .
$$

Theorem 1.2. We consider the differential system (1.4) where the functions $a(t), b(t), c(t)$ and $h(t, x)$ are $C^{2}$ and $T-$ periodic in the variable $t$. Assume that the functions of (1.6) are T-periodic in the variable $t$. Then for every simple zero $\left(x_{0}^{*}, y_{0}^{*}\right)$ of one of polynomial systems (3.14)-(3.21) in the variables $x_{0}$ and $y_{0}$ given in Appendix 3 , the differential system (1.4) has a T-periodic solution $(x(t), y(t))$ such that $(x(0), y(0))$ is close to $\left(x_{0}^{*}, y_{0}^{*}\right)$.

Theorem 1.1 is proved in section 2.

The proof of Theorem 1.2 is similar to the proof of Theorem 1.1 and we do not provide it here, otherwise this paper will duplicate the number of pages.

These proofs are based in the averaging theory and in different rescalings of the functions $a(t), b(t), c(t)$ and $h(t, x)$, and of the variables $x$ and $y$.

In section 3 we provide differential systems satisfying all the assumptions of Theorems 1.1 and 1.2, i.e. there are differential systems for which every polynomial system from (3.6) up to (3.21) provides limit cycles of those systems.

In Appendix 1 we recall the basic result of the averaging theory that we need for proving our results.

\section{Proof of Theorem 1.1}

We do the following rescaling of the functions and variables which appear in the differential system (1.4)

$$
\begin{aligned}
x & =X, \\
y & =\varepsilon^{m_{2}} Y, \\
a(t) & =\varepsilon^{n_{1}} A(t), \\
b(t) & =\varepsilon^{n_{2}} B(t), \\
c(t) & =\varepsilon^{n_{3}} C(t), \\
h(t, x) & =\varepsilon^{n_{4}} H(t, X),
\end{aligned}
$$

where $\varepsilon>0$ is a small parameter, then the differential system (1.4) becomes

$$
\begin{aligned}
X^{\prime}= & \varepsilon^{m_{2}} Y, \\
Y^{\prime}= & -\varepsilon^{n_{3}} C(t) Y-\varepsilon^{n_{1}-m_{2}} A(t) X \\
& -\varepsilon^{n_{2}-m_{2}} B(t) X^{3}+\varepsilon^{n_{4}-m_{2}} H(t, X),
\end{aligned}
$$

where we assume $0 \leq n_{3}, 0 \leq m_{2} \leq n_{1}, m_{2} \leq n_{2}, m_{2} \leq n_{4}$.

We distinguish the following two cases with their corresponding subcases

Case $\mathbf{I}: m_{2}=1$ and $n_{3}=0$.

Case II : $m_{2}=1$ and $n_{3}=1$.

We split the cases I and II into the following eight subcases

( $\alpha .1) n_{1}-m_{2}=0, n_{2}-m_{2}=0, n_{4}-m_{2}=0$,

( $\alpha .2) n_{1}-m_{2}=0, n_{2}-m_{2}=0, n_{4}-m_{2}=1$ ，

( $\alpha .3) n_{1}-m_{2}=0, n_{2}-m_{2}=1, n_{4}-m_{2}=0$,

( $\alpha .4) n_{1}-m_{2}=1, n_{2}-m_{2}=0, n_{4}-m_{2}=0$,

( $\alpha .5) n_{1}-m_{2}=0, n_{2}-m_{2}=1, n_{4}-m_{2}=1$,

( $\alpha .6) n_{1}-m_{2}=1, n_{2}-m_{2}=0, n_{4}-m_{2}=1$,

( $\alpha .7) n_{1}-m_{2}=1, n_{2}-m_{2}=1, n_{4}-m_{2}=0$,

( $\alpha .8) n_{1}-m_{2}=1, n_{2}-m_{2}=1, n_{4}-m_{2}=1$,

where $\alpha \in\{\mathbf{I}, \mathbf{I I}\}$.

We shall prove Theorem 1.1 statement by statement. 
We assume that the following functions are $T$-periodic in the variable $t$

$$
\begin{array}{ll}
K(t)=\int_{0}^{t} C(s) d s, & M(t)=\int_{0}^{t} A(s) e^{K(s)} d s, \\
N(t) & =\int_{0}^{t} B(s) e^{K(s)} d s, \quad G\left(t, X_{0}\right)=\int_{0}^{t} H\left(s, X_{0}\right) e^{K(s)} d s .
\end{array}
$$

Proof of Theorem 1.1 for the polynomial system (3.6). For the case (I.1), i.e. for $n_{3}=0, m_{2}=n_{1}=n_{2}=n_{4}=1$, the differential system (2.2) writes

$$
\begin{aligned}
& X^{\prime}=\varepsilon Y, \\
& Y^{\prime}=-C(t) Y-A(t) X-B(t) X^{3}+H(t, X) .
\end{aligned}
$$

This system is in the normal form (3.1) for applying the averaging theory described in Appendix 1. More precisely, we have $x=(X, Y)$,

$$
F_{0}(t, x)=\left(\begin{array}{c}
0 \\
-C(t) Y-A(t) X-B(t) X^{3}+H(t, X)
\end{array}\right), \quad F_{1}(t, x)=\left(\begin{array}{c}
Y \\
0
\end{array}\right) \text { and } F_{2}(t, x)=\left(\begin{array}{c}
0 \\
0
\end{array}\right) .
$$

Solving the differential system (3.2) associated to our system (2.3) we obtain the T-periodic solutions

$$
(X(t), Y(t))=\left(X_{0},\left(Y_{0}-X_{0} M(t)-X_{0}^{3} N(t)+G\left(t, X_{0}\right)\right) e^{-K(t)}\right)
$$

for all $\left(X_{0}, Y_{0}\right) \in \mathbb{R}^{2} \backslash\{(0,0)\}$. Now taking $z=\left(X_{0}, Y_{0}\right)$ and solving the variational differential equation (3.3) corresponding to our system (2.3) we obtain the fundamental matrix

$$
M_{\mathrm{Z}}(t)=\left(\begin{array}{cc}
1 & 0 \\
\left(-M(t)-3 X_{0}^{2} N(t)+G_{x}\left(t, X_{0}\right)\right) e^{-K(t)} & e^{-K(t)}
\end{array}\right) .
$$

We compute the average function $\mathcal{F}(z)=\left(\mathcal{F}_{1}\left(X_{0}, Y_{0}\right), \mathcal{F}_{2}\left(X_{0}, Y_{0}\right)\right)$ defined in $(3.4)$ and we get

$$
\begin{aligned}
& \mathcal{F}_{1}=-X_{0}^{3} \int_{0}^{T} N(t) e^{-K(t)} d t-X_{0} \int_{0}^{T} M(t) e^{-K(t)} d t+\int_{0}^{T} G\left(t, X_{0}\right) e^{-K(t)} d t+Y_{0} \int_{0}^{T} e^{-K(t)} d t, \\
& \mathcal{F}_{2}=-3 X_{0}^{5} \int_{0}^{T} N(t)^{2} e^{-K(t)} d t-X_{0}^{3} \int_{0}^{T} N(t)\left(4 M(t)-G_{x}\left(t, X_{0}\right)\right) e^{-K(t)} d t+ \\
& 3 X_{0}^{2} Y_{0} \int_{0}^{T} N(t) e^{-K(t)} d t+3 X_{0}^{2} \int_{0}^{T} N(t) G\left(t, X_{0}\right) e^{-K(t)} d t-X_{0} \int_{0}^{T} M(t) . \\
& \left(M(t)-G\left(t, X_{0}\right)\right) e^{-K(t)} d t+\int_{0}^{T} G\left(t, X_{0}\right)\left(M(t)-G_{x}\left(t, X_{0}\right)\right) e^{-K(t)} d t \\
& +Y_{0}^{T}\left(M(t)-G_{x}\left(t, X_{0}\right)\right) e^{-K(t)} d t .
\end{aligned}
$$

By Theorem 3.1 the differential system (2.3) has a periodic solution $(X(t, \varepsilon), Y(t, \varepsilon))$ such that $(X(0, \varepsilon), Y(0, \varepsilon)) \rightarrow$ $\left(X_{0}^{*}, Y_{0}^{*}\right)$ when $\varepsilon \rightarrow 0$, for each zero $\left(X_{0}^{*}, Y_{0}^{*}\right)$ of the system $\mathcal{F}_{1}=\mathcal{F}_{2}=0$, whose Jacobian is different from zero, i.e. $\left(X_{0}^{*}, Y_{0}^{*}\right)$ is a simple zero of the system $\mathcal{F}_{1}=\mathcal{F}_{2}=0$. Going back to the differential system (1.4) through the rescalings (2.1) the polynomial system $\mathcal{F}_{1}=\mathcal{F}_{2}=0$ in the variables $X_{0}$ and $Y_{0}$ becomes the polynomial system (3.6) in the variables $x_{0}$ and $y_{0}$. Consequently the theorem is proved for system (3.6). 
Proof of Theorem 1.1 for the polynomial system (3.7). For the case (I.2), i.e. for $n_{3}=0, m_{2}=n_{1}=n_{2}=1, n_{4}=2$, the differential system (2.2) writes

$$
\begin{aligned}
& X^{\prime}=\varepsilon Y \\
& Y^{\prime}=-C(t) Y-A(t) X-B(t) X^{3}+\varepsilon H(t, X) .
\end{aligned}
$$

This system is in the normal form (3.1) for applying the averaging theory described in Appendix 1.

Solving the differential system (3.2) we obtain the T-periodic solutions

$$
(X(t), Y(t))=\left(X_{0},\left(-X_{0} M(t)-X_{0}^{3} N(t)+Y_{0}\right) e^{-K(t)}\right),
$$

for all $\left(X_{0}, Y_{0}\right) \in \mathbb{R}^{2} \backslash\{(0,0)\}$. Now taking $z=\left(X_{0}, Y_{0}\right)$ and solving the variational differential equation (3.3) we obtain the fundamental matrix

$$
M_{\mathbf{Z}}(t)=\left(\begin{array}{cc}
1 & 0 \\
\left(-M(t)-3 X_{0}^{2} N(t)\right) e^{-K(t)} & e^{-K(t)}
\end{array}\right) .
$$

We compute the average function $\mathcal{F}(\mathrm{z})=\left(\mathcal{F}_{1}\left(X_{0}, Y_{0}\right), \mathcal{F}_{2}\left(X_{0}, Y_{0}\right)\right)$ defined in (3.4) and we get

$$
\begin{aligned}
\mathcal{F}_{1}= & -X_{0}^{3} \int_{0}^{T} N(t) e^{-K(t)} d t-X_{0} \int_{0}^{T} M(t) e^{-K(t)} d t+Y_{0} \int_{0}^{T} e^{-K(t)} d t \\
\mathcal{F}_{2}= & -3 X_{0}^{5} \int_{0}^{T} N(t)^{2} e^{-K(t)} d t-4 X_{0}^{3} \int_{0}^{T} N(t) M(t) e^{-K(t)} d t+3 X_{0}^{2} Y_{0} \int_{0}^{T} N(t) . \\
& e^{-K(t)} d t-X_{0} \int_{0}^{T} M(t)^{2} e^{-K(t)} d t+\int_{0}^{T} H\left(t, X_{0}\right) e^{K(t)} d t+Y_{0} \int_{0}^{T} M(t) e^{-K(t)} d t .
\end{aligned}
$$

By Theorem 3.1 the differential system (2.4) has a periodic solution $(X(t, \varepsilon), Y(t, \varepsilon))$ such that $(X(0, \varepsilon), Y(0, \varepsilon)) \rightarrow$ $\left(X_{0}^{*}, Y_{0}^{*}\right)$ when $\varepsilon \rightarrow 0$, for each zero $\left(X_{0}^{*}, Y_{0}^{*}\right)$ of the system $\mathcal{F}_{1}=\mathcal{F}_{2}=0$, whose Jacobian is different from zero. Going back to the differential system (1.4) through the rescalings (2.1) the theorem follows for system (3.7).

Proof of Theorem 1.1 for the polynomial system (3.8). For the case (I.3), i.e. for $n_{3}=0, m_{2}=n_{1}=n_{4}=1, n_{2}=2$, the differential system (2.2) writes

$$
\begin{aligned}
& X^{\prime}=\varepsilon Y, \\
& Y^{\prime}=-C(t) Y-A(t) X+H(t, X)-\varepsilon B(t) X^{3} .
\end{aligned}
$$

This system is in the normal form (3.1) for applying the averaging theory. Solving the differential system (3.2) we obtain the $T$-periodic solutions

$$
(X(t), Y(t))=\left(X_{0},\left(-X_{0} M(t)+G\left(t, X_{0}\right)+Y_{0}\right) e^{-K(t)}\right),
$$

for all $\left(X_{0}, Y_{0}\right) \in \mathbb{R}^{2} \backslash\{(0,0)\}$. Now in a similar way to the proof of the previous two systems (3.6) and (3.7) we obtain the fundamental matrix

$$
M_{\mathbf{Z}}(t)=\left(\begin{array}{cc}
1 & 0 \\
\left(-M(t)+G_{x}\left(t, X_{0}\right)\right) e^{-K(t)} & e^{-K(t)}
\end{array}\right) .
$$


Computing the average function $\mathcal{F}(\mathrm{z})=\left(\mathcal{F}_{1}\left(X_{0}, Y_{0}\right), \mathcal{F}_{2}\left(X_{0}, Y_{0}\right)\right)$ we get

$$
\begin{aligned}
\mathcal{F}_{1}= & -X_{0} \int_{0}^{T} M(t) e^{-K(t)} d t+\int_{0}^{T} G\left(t, X_{0}\right) e^{-K(t)} d t+Y_{0} \int_{0}^{T} e^{-K(t)} d t, \\
\mathcal{F}_{2}= & -X_{0}^{3} \int_{0}^{T} B(t) e^{K(t)} d t-X_{0} \int_{0}^{T} M(t)\left(M(t)-G_{x}\left(t, X_{0}\right)\right) e^{-K(t)} d t+\int_{0}^{T} G\left(t, X_{0}\right) \\
& \left(M(t)-G_{x}\left(t, X_{0}\right)\right) e^{-K(t)} d t+Y_{0} \int_{0}^{T}\left(M(t)-G_{x}\left(t, X_{0}\right)\right) e^{-K(t)} d t .
\end{aligned}
$$

Now the proof of the theorem for the polynomial system (3.8) follows as the proofs for the previous polynomial systems (3.6) and (3.7).

Proof of Theorem 1.1 for the polynomial system (3.9). For the case (I.4), i.e. for $n_{3}=0, m_{2}=n_{2}=n_{4}=1, n_{1}=2$, the differential system (2.2) writes

$$
\begin{aligned}
& X^{\prime}=\varepsilon Y, \\
& Y^{\prime}=-C(t) Y-B(t) X^{3}+H(t, X)-\varepsilon A(t) X .
\end{aligned}
$$

This system is in the normal form (3.1) for applying the averaging theory. Solving the differential system (3.2) we obtain the $T$-periodic solutions

$$
(X(t), Y(t))=\left(X_{0},\left(-X_{0}^{3} N(t)+G\left(t, X_{0}\right)+Y_{0}\right) e^{-K(t)}\right),
$$

for all $\left(X_{0}, Y_{0}\right) \in \mathbb{R}^{2} \backslash\{(0,0)\}$. Solving the variational differential equation (3.3) we obtain the fundamental matrix

$$
M_{\mathrm{z}}(t)=\left(\begin{array}{cc}
1 & 0 \\
\left(-3 X_{0}^{2} N(t)+G_{x}\left(t, X_{0}\right)\right) e^{-K(t)} & e^{-K(t)}
\end{array}\right) .
$$

We compute the average function and we obtain

$$
\begin{aligned}
\mathcal{F}_{1}= & -X_{0}^{3} \int_{0}^{T} N(t) e^{-K(t)} d t+\int_{0}^{T} G\left(t, X_{0}\right) e^{-K(t)} d t+Y_{0} \int_{0}^{T} e^{-K(t)} d t \\
\mathcal{F}_{2}= & -3 X_{0}^{5} \int_{0}^{T} N(t)^{2} e^{-K(t)} d t+X_{0}^{3} \int_{0}^{T} N(t) G_{x}\left(t, X_{0}\right) e^{-K(t)} d t+3 X_{0}^{2} Y_{0} \int_{0}^{T} N(t) \\
& e^{-K(t)} d t+3 X_{0}^{2} \int_{0}^{T} N(t) G\left(t, X_{0}\right) e^{-K(t)} d t-X_{0} \int_{0}^{T} A(t) e^{K(t)} d t-\int_{0}^{T} G\left(t, X_{0}\right) \\
& G_{x}\left(t, X_{0}\right) e^{-K(t)} d t-Y_{0} \int_{0}^{T} G_{x}\left(t, X_{0}\right) e^{-K(t)} d t .
\end{aligned}
$$

As in the proofs of the theorem for the previous polynomial systems it follows the proof for the polynomial system (3.9).

Proof of Theorem 1.1 for the polynomial system (3.10). For the case (I.5), i.e. for $n_{3}=0, m_{2}=n_{1}=1, n_{2}=n_{4}=2$, the differential system (2.2) writes

$$
\begin{aligned}
& X^{\prime}=\varepsilon Y, \\
& Y^{\prime}=-C(t) Y-A(t) X+\varepsilon H(t, X)-\varepsilon B(t) X^{3} .
\end{aligned}
$$


We can apply the averaging theory to system (2.5). Solving the differential system (3.2) we obtain the T-periodic solutions

$$
(X(t), Y(t))=\left(X_{0},\left(-X_{0} M(t)+Y_{0}\right) e^{-K(t)}\right),
$$

for all $\left(X_{0}, Y_{0}\right) \in \mathbb{R}^{2} \backslash\{(0,0)\}$. Solving the variational differential equation (3.3) we obtain the fundamental matrix

$$
M_{\mathrm{Z}}(t)=\left(\begin{array}{cc}
1 & 0 \\
-M(t) e^{-K(t)} & e^{-K(t)}
\end{array}\right) .
$$

We compute the average function

$$
\begin{aligned}
\mathcal{F}_{1}= & -X_{0} \int_{0}^{T} M(t) e^{-K(t)} d t+Y_{0} \int_{0}^{T} e^{-K(t)} d t \\
\mathcal{F}_{2}= & -X_{0}^{3} \int_{0}^{T} B(t) e^{K(t)} d t-X_{0} \int_{0}^{T} M(t)^{2} e^{-K(t)} d t+\int_{0}^{T} H\left(t, X_{0}\right) e^{K(t)} d t+ \\
& Y_{0} \int_{0}^{T} M(t) e^{-K(t)} d t .
\end{aligned}
$$

As in the proofs of the theorem for the previous polynomial systems it follows the proof for the polynomial system (3.10).

Proof of Theorem 1.1 for the polynomial system (3.11). For the case (I.6), i.e. for $n_{3}=0, m_{2}=n_{2}=1, n_{1}=n_{4}=2$, the differential system (2.2) writes

$$
\begin{aligned}
& X^{\prime}=\varepsilon Y, \\
& Y^{\prime}=-C(t) Y-B(t) X^{3}-\varepsilon A(t) X+\varepsilon H(t, X) .
\end{aligned}
$$

We apply the averaging theory, so solving the differential system (3.2) we obtain the $T$ - periodic solutions

$$
(X(t), Y(t))=\left(X_{0},\left(-X_{0}^{3} N(t)+Y_{0}\right) e^{-K(t)}\right),
$$

for all $\left(X_{0}, Y_{0}\right) \in \mathbb{R}^{2} \backslash\{(0,0)\}$. Solving the variational differential equation (3.3) we obtain the fundamental matrix

$$
M_{\mathrm{Z}}(t)=\left(\begin{array}{cc}
1 & 0 \\
-3 X_{0}^{2} N(t) e^{-K(t)} & e^{-K(t)}
\end{array}\right) .
$$

And we obtain the corresponding average function

$$
\begin{aligned}
\mathcal{F}_{1}= & -X_{0}^{3} \int_{0}^{T} N(t) e^{-K(t)} d t+Y_{0} \int_{0}^{T} e^{-K(t)} d t \\
\mathcal{F}_{2}= & -3 X_{0}^{5} \int_{0}^{T} N(t)^{2} e^{-K(t)} d t+3 X_{0}^{2} Y_{0} \int_{0}^{T} N(t) e^{-K(t)} d t-X_{0} \int_{0}^{T} A(t) e^{K(t)} d t+ \\
& \int_{0}^{T} H\left(t, X_{0}\right) e^{K(t)} d t .
\end{aligned}
$$

As in the proofs of the theorem for the previous polynomial systems it follows the proof for the polynomial system (3.11). 
Proof of Theorem 1.1 for the polynomial system (3.12). For the case (I.7), i.e. for $n_{3}=0, m_{2}=n_{4}=1, n_{1}=n_{2}=2$, the differential system (2.2) writes

$$
\begin{aligned}
& X^{\prime}=\varepsilon Y, \\
& Y^{\prime}=-C(t) Y+H(t, X)-\varepsilon A(t) X-\varepsilon B(t) X^{3} .
\end{aligned}
$$

Solving the differential system (3.2) we obtain the $T$-periodic solutions

$$
(X(t), Y(t))=\left(X_{0},\left(G\left(t, X_{0}\right)+Y_{0}\right) e^{-K(t)}\right),
$$

for all $\left(X_{0}, Y_{0}\right) \in \mathbb{R}^{2} \backslash\{(0,0)\}$. Now solving the variational differential equation (3.3) we obtain the fundamental matrix

$$
M_{\mathrm{Z}}(t)=\left(\begin{array}{cc}
1 & 0 \\
G_{x}\left(t, X_{0}\right) e^{-K(t)} & e^{-K(t)}
\end{array}\right) .
$$

We compute the average function and we obtain

$$
\begin{aligned}
\mathcal{F}_{1}= & \int_{0}^{T} G\left(t, X_{0}\right) e^{-K(t)} d t+Y_{0} \int_{0}^{T} e^{-K(t)} d t \\
\mathcal{F}_{2}= & -X_{0}^{3} \int_{0}^{T} B(t) e^{K(t)} d t-X_{0} \int_{0}^{T} A(t) e^{K(t)} d t-\int_{0}^{T} G\left(t, X_{0}\right) G_{x}\left(t, X_{0}\right) e^{-K(t)} d t- \\
& Y_{0} \int_{0}^{T} G_{x}\left(t, X_{0}\right) e^{-K(t)} d t .
\end{aligned}
$$

As in the proofs of the theorem for the previous polynomial systems it follows the proof for the polynomial system (3.12).

Proof of Theorem 1.1 for the polynomial system (3.13). For the case (I.8), i.e. for $n_{3}=0, m_{2}=1, n_{1}=n_{2}=n_{4}=2$, the differential system (2.2) writes

$$
\begin{aligned}
& X^{\prime}=\varepsilon Y, \\
& Y^{\prime}=-C(t) Y-\varepsilon A(t) X-\varepsilon B(t) X^{3}+\varepsilon H(t, X) .
\end{aligned}
$$

Solving the differential system (3.2) we obtain the $T$-periodic solutions

$$
(X(t), Y(t))=\left(X_{0}, Y_{0} e^{-K(t)}\right),
$$

for all $\left(X_{0}, Y_{0}\right) \in \mathbb{R}^{2} \backslash\{(0,0)\}$. Solving the variational differential equation (3.3) we obtain the fundamental matrix

$$
M_{\mathrm{Z}}(t)=\left(\begin{array}{cc}
1 & 0 \\
0 & e^{-K(t)}
\end{array}\right) .
$$

We compute the average function and we get

$$
\begin{aligned}
& \mathcal{F}_{1}=Y_{0} \int_{0}^{T} e^{-K(t)} d t \\
& \mathcal{F}_{2}=-X_{0}^{3} \int_{0}^{T} B(t) e^{K(t)} d t-X_{0} \int_{0}^{T} A(t) e^{K(t)} d t+\int_{0}^{T} H\left(t, X_{0}\right) e^{K(t)} d t .
\end{aligned}
$$

As in the proofs of the theorem for the previous polynomial systems it follows the proof for the polynomial system (3.13).

This completes the proof of Theorem 1.1. 


\section{Examples}

In this section we provide examples of each one of the statements of Theorems 1.1 and 1.2.

Example (1.1) : Consider the differential system (1.4) with $a(t)=-\left(\frac{2}{3}+\frac{1}{3} \cos t\right) \sin t \cos t, b(t)=\left(\frac{2}{3}+\right.$ $\left.\frac{1}{3} \cos t\right) \cos t, \mathbf{c}(\mathbf{t})=\frac{\sin \mathbf{t}}{2+\cos \mathbf{t}}$ and $h(t, x)=x\left(\frac{2}{3}+\frac{1}{3} \cos t\right) \cos t$. These functions are $2 \pi$-periodic in the variable $t$. Then we obtain the functions $k(t)=\int_{0}^{t} c(s) d s=-\ln \left(\frac{2+\cos t}{3}\right), g(t, x)=\int_{0}^{t} h(s, x) e^{k(s)} d s=x \sin t$, $m(t)=\int_{0}^{t} a(s) e^{k(s)} d s=-\frac{1}{2}+\frac{1}{2} \cos ^{2} t$ and $n(t)=\int_{0}^{t} b(s) e^{k(s)} d s=\sin t$, are $2 \pi$-periodic in the variable $t$. Now the system (3.6) of Theorem 1.1 becomes

$$
\frac{1}{3} x_{0}+\frac{4}{3} y_{0}=0, \quad-2 \mathbf{x}_{0}^{5}+\frac{8}{3} x_{0}^{3}-\frac{19}{24} x_{0}-\frac{1}{3} y_{0}=0
$$

This system has five solutions:

$$
(0,0), \quad s_{ \pm}= \pm\left(\frac{1}{6} \sqrt{24-3 \sqrt{13}},-\frac{1}{24} \sqrt{24-3 \sqrt{13}}\right), \quad r_{ \pm}= \pm\left(\frac{1}{6} \sqrt{24+3 \sqrt{13}},-\frac{1}{24} \sqrt{24+3 \sqrt{13}}\right) .
$$

We omit the solution $(0,0)$, which corresponds to an equilibrium point. The differential system (1.4) for this example is invariant under the symmetry $(x, y) \rightarrow(-x,-y)$. So since the Jacobian (3.5) is $\frac{26}{27}-\frac{16}{27} \sqrt{13}$ for the second and third solution, and $\frac{26}{27}+\frac{16}{27} \sqrt{13}$ for the fourth and fifth solution, the differential system (1.4) for $\varepsilon \neq 0$ sufficiently small has two periodic solutions $(x(t, \varepsilon), y(t, \varepsilon))$ such that $(x(0, \varepsilon), y(0, \varepsilon))$ tends to $s_{+}$or $r_{+}$when $\varepsilon \rightarrow 0$.

Example (1.2) : Consider the differential system (1.4) with $a(t)=(1+(\sin t) / 2) \sin t, b(t)=(1+(\sin t) / 2) \cos t, c(t)=$ $-\cos t /(2+\sin t), h(t, x)=x$. These functions are $2 \pi$-periodic in the variable $t$. Then we get the functions $k(t)=\int_{0}^{t} c(s) d s=-\ln ((2+\sin t) / 2), m(t)=\int_{0}^{t} a(s) e^{k(s)} d s=1-\cos t$ and $n(t)=\int_{0}^{t} b(s) e^{k(s)} d s=\sin t$, which are $2 \pi$-periodic in the variable $t$. Now computing the polynomial system (3.7) of Theorem 1.1 we obtain the system

$$
-\frac{1}{2} x_{0}^{3}-2 x_{0}+2 y_{0}=0, \quad-3 x_{0}^{5}-2 x_{0}^{3}+\frac{3}{2} x_{0}^{2} y_{0}-3 x_{0}+\frac{4}{3} \sqrt{3} x_{0}+2 y_{0}=0 .
$$

This system has only two real solutions different from $(0,0)$ :

$$
s_{ \pm}= \pm\left(\frac{\sqrt{42}}{21}(-42+56 \sqrt{3})^{1 / 4}, \frac{\sqrt{42}}{882}(-42+56 \sqrt{3})^{3 / 4}+\frac{\sqrt{42}}{21}(-42+56 \sqrt{3})^{1 / 4}\right) .
$$

The differential system (1.4) for this example is invariant under the symmetry $(x, y) \rightarrow(-x,-y)$. So since the Jacobian (3.5) is $32 \sqrt{3} / 3-8$ for the second and third solution, the differential system (1.4) for $\varepsilon \neq 0$ sufficiently small has one periodic solution $(x(t, \varepsilon), y(t, \varepsilon))$ such that $(x(0, \varepsilon), y(0, \varepsilon))$ tends to $s_{+}$when $\varepsilon \rightarrow 0$.

Example (1.3) : Consider the differential system (1.4) with $a(t)=(1+(\sin t) / 2) \cos t, b(t)=\cos t, c(t)=$ $-\sin t /(2+\cos t), h(t, x)=(x+1)(1+(\sin t) / 2) \sin t$. These functions are $2 \pi$-periodic in the variable $t$. Then we obtain the functions $k(t)=\int_{0}^{t} c(s) d s=-\ln ((2+\cos t) / 3), m(t)=\int_{0}^{t} a(s) e^{k(s)} d s=\sin t$ and $g(t, x)=$ 
$\int_{0}^{t} h(s, x) e^{k(s)} d s=-x \cos t-\cos t+x+1$, which are $2 \pi$-periodic in the variable $t$. Now the polynomial system (3.8) of Theorem 1.1 becomes

$$
\frac{3}{2} x_{0}+2 y_{0}+2=0, \quad-3 x_{0}-\frac{3}{2} y_{0}-\frac{5}{2}=0 .
$$

This system has the unique solution $z=(-8 / 15,-3 / 5)$. Since the Jacobian (3.5), for this solution is $15 / 4$, the differential system (1.4) for $\varepsilon \neq 0$ sufficiently small has one periodic solution $(x(t, \varepsilon), y(t, \varepsilon))$ such that $(x(0, \varepsilon), y(0, \varepsilon))$ tends to $s$ when $\varepsilon \rightarrow 0$.

Example (1.4) : Consider the differential system (1.4) with $a(t)=\sin t+\cos t, b(t)=(2+\cos t) \cos t / 3$, $\mathbf{c}(\mathbf{t})=\sin \mathbf{t} /(2+\cos \mathbf{t}), h(t, x)=x(2+\cos t) \sin t / 3$. These functions are $2 \pi$-periodic in the variable $t$. Then we compute the functions $k(t)=\int_{0}^{t} c(s) d s=-\ln ((2+\cos t) / 3), g(t, x)=\int_{0}^{t} h(s, x) e^{k(s)} d s=x-x \cos t$ and $n(t)=\int_{0}^{t} b(s) e^{k(s)} d s=\sin t$, which are $2 \pi$-periodic in the variable $t$. For this system the polynomial system (3.9) of Theorem 1.1 is

$$
x_{0}+\frac{4}{3} y_{0}=0, \quad-2 x_{0}^{5}-\frac{22}{3} x_{0}+4 \sqrt{3} x_{0}-y_{0}=0 .
$$

This system has only two non-zero real solutions

$$
s_{ \pm}= \pm\left(\frac{\sqrt{3}}{6}(-474+288 \sqrt{3})^{1 / 4},-\frac{\sqrt{3}}{8}(-474+288 \sqrt{3})^{1 / 4}\right) .
$$

The differential system (1.4) for this example is invariant under the symmetry $(x, y) \rightarrow(-x,-y)$. So since the Jacobian (3.5) is $-316 / 9+64 \sqrt{3} / 3$ for both solutions, the differential system (1.4) for $\varepsilon \neq 0$ sufficiently small has one periodic solution $(x(t, \varepsilon), y(t, \varepsilon))$ such that $(x(0, \varepsilon), y(0, \varepsilon))$ tends to $s_{+}$when $\varepsilon \rightarrow 0$.

Example (1.5) : Consider the differential system (1.4) with $a(t)=3\left(1+\sin ^{2} t\right) \sin t, b(t)=\sin ^{2} t(1+\cos t)$, $c(t)=-2 \sin t \cos t /\left(1+\sin ^{2} t\right)$ and $h(t, x)=\left(x^{3}+1\right) \sin ^{2} t$. These functions are $2 \pi$-periodic in the variable $t$. Then we obtain the functions $k(t)=\int_{0}^{t} c(s) d s=-\ln \left(1+\sin ^{2} t\right)$ and $m(t)=\int_{0}^{t} a(s) e^{k(s)} d s=3-3 \cos t$, which are $2 \pi$-periodic in the variable $t$. For this system the polynomial system (3.10) of Theorem 1.1 is

$$
-9 x_{0}+3 y_{0}=0, \quad-\frac{153}{4} x_{0}+9 y_{0}-\sqrt{2}+2=0 .
$$

This system has the unique solution $s=((8-4 \sqrt{2}) / 45,(8-4 \sqrt{2}) / 15)$. Since the Jacobian (3.5) for this solution is $135 / 4$, the differential system (1.4) for $\varepsilon \neq 0$ sufficiently small has one periodic solution $(x(t, \varepsilon), y(t, \varepsilon))$ such that $(x(0, \varepsilon), y(0, \varepsilon))$ tends to $s$ when $\varepsilon \rightarrow 0$.

Example (1.6) : Consider the differential system (1.4) with $a(t)=\sin ^{2} t, b(t)=\cos t\left(1+\sin ^{2} t\right), c(t)=$ $-2 \sin t \cos t /\left(1+\sin ^{2} t\right)$ and $h(t, x)=(x+1) \sin ^{2} t$. These functions are $2 \pi$-periodic in the variable $t$. Then we compute the functions $k(t)=\int_{0}^{t} c(s) d s=-\ln \left(1+\sin ^{2} t\right)$ and $n(t)=\int_{0}^{t} b(s) e^{k(s)} d s=\sin t$, which are $2 \pi$-periodic in the variable $t$. Here the polynomial system (3.11) of Theorem 1.1 becomes

$$
3 y_{0}=0, \quad-\frac{21}{4} x_{0}^{5}+2-\sqrt{2}=0 .
$$

This system has the unique real solution $s=\left((-777924 \sqrt{2}+1555848)^{1 / 5} / 21,0\right)$. Since the Jacobian (3.5) for this solution is $5(-777924 \sqrt{2}+1555848)^{4 / 5} / 12348$, the differential system (1.4) for $\varepsilon \neq 0$ sufficiently small has one periodic solution $(x(t, \varepsilon), y(t, \varepsilon))$ such that $(x(0, \varepsilon), y(0, \varepsilon))$ tends to $s$ when $\varepsilon \rightarrow 0$. 
Example (1.7) : Consider the differential system (1.4) with $a(t)=\sin ^{2} t, b(t)=\cos t\left(1+\sin ^{2} t\right), c(t)=$ $-2 \sin t \cos t /\left(1+\sin ^{2} t\right)$ and $h(t, x)=(x+1)\left(1+\sin ^{2} t\right) \cos t$. These functions are $2 \pi$-periodic in the variable

$t$. Then we get the functions $k(t)=\int_{0}^{t} c(s) d s=-\ln \left(1+\sin ^{2} t\right)$ and $g(t, x)=\int_{0}^{t} h(s, x) e^{k(s)} d s=(x+1) \sin t$, which are $2 \pi$-periodic in the variable $t$. Now the polynomial system (3.12) of Theorem 1.1 is

$$
3 y_{0}=0, \quad-\frac{15}{4} x_{0}+\sqrt{2} x_{0}-\frac{7}{4}=0 .
$$

This system has the unique solution $s=(7 /(4 \sqrt{2}-15), 0)$. Since the Jacobian (3.5) for this solution is $-3(-15+$ $4 \sqrt{2}) / 4$, the differential system (1.4) for $\varepsilon \neq 0$ sufficiently small has one periodic solution $(x(t, \varepsilon), y(t, \varepsilon))$ such that $(x(0, \varepsilon), y(0, \varepsilon))$ tends to $s$ when $\varepsilon \rightarrow 0$.

Example (1.8) : Consider the differential system (1.4) with $a(t)=2 \cos t, b(t)=\cos ^{2} t, c(t)=\sin t /(2+\cos t)$ and $h(t, x)=x \cos t \sin ^{2} t$. These functions are $2 \pi$-periodic in the variable $t$. Then we compute the function $k(t)=\int_{0}^{t} c(s) d s=-\ln ((2+\cos t) / 3)$, which is $2 \pi$-periodic in the variable $t$. For this differential system the polynomial system (3.13) of Theorem 1.1 becomes

$$
y_{0}=0, \quad-8 \sqrt{3} x_{0}^{3}+12 x_{0}^{3}+20 \sqrt{3} x_{0}-33 x_{0}=0 .
$$

It has the two non-zero solutions $s_{ \pm}= \pm \sqrt{7-2 \sqrt{3}} / 2$, whose Jacobians (3.5) are $40 \sqrt{3}-66$. Since system (1.4) for this example is invariant under the symmetry $(x, y) \rightarrow(-x,-y)$, it has for $\varepsilon \neq 0$ sufficiently small one periodic solution $(x(t, \varepsilon), y(t, \varepsilon))$ such that $(x(0, \varepsilon), y(0, \varepsilon))$ tends to $s_{+}$when $\varepsilon \rightarrow 0$.

Example (2.1) : Consider the differential system (1.4) with $a(t)=\sin t, b(t)=2 \cos t, c(t)=2 \sin t+\cos t$ and $h(t, x)=x \sin t$. These functions are $2 \pi$-periodic in the variable $t$. Then we obtain the functions $g(t, x)=$ $\int_{0}^{t} h(s, x) d s=x-x \cos t, m(t)=\int_{0}^{t} a(s) d s=1-\cos t$ and $n(t)=\int_{0}^{t} b(s) d s=2 \sin t$, which are $2 \pi$-periodic in the variable $t$. For this differential system the polynomial differential system (3.14) of Theorem 1.2 is

$$
2 y_{0}=0, \quad-12 x_{0}^{5}+4 x_{0}^{3}=0 .
$$

This system has the two non-zero solutions $s_{ \pm}= \pm(1 / \sqrt{3}, 0)$. Since the Jacobian (3.5) is $16 / 3$ for both solution, and system (1.4) for this example is invariant under the symmetry $(x, y) \rightarrow(-x,-y)$ it has for $\varepsilon \neq 0$ sufficiently small one periodic solution $(x(t, \varepsilon), y(t, \varepsilon))$ such that $(x(0, \varepsilon), y(0, \varepsilon))$ tends to $s_{+}$when $\varepsilon \rightarrow 0$.

Example (2.2) : Consider the differential system (1.4) with $a(t)=-\sin t, b(t)=\cos t, c(t)=\sin t$ and $h(t, x)=$ $x$. These functions are $2 \pi$-periodic in the variable $t$. Then we compute the functions $m(t)=\int_{0}^{t} a(s) d s=-1+\cos t$ and $n(t)=\int_{0}^{t} b(s) d s=\sin t$, which are $2 \pi$-periodic in the variable $t$. For this differential system the polynomial system (3.15) of Theorem 1.2 becomes

$$
2 x_{0}+2 y_{0}=0, \quad-3 x_{0}^{5}+x_{0}^{3}-x_{0}-2 y_{0}=0 .
$$

This system has the two non-zero real solutions $s_{ \pm}= \pm(\sqrt{6+6 \sqrt{13}} / 6,-\sqrt{6+6 \sqrt{13}} / 6)$. Since the Jacobian (3.5) is $(26+2 \sqrt{13}) / 3$ for both solution, and system (1.4) for this example is invariant under the symmetry $(x, y) \rightarrow(-x,-y)$ it has for $\varepsilon \neq 0$ sufficiently small one periodic solutions $(x(t, \varepsilon), y(t, \varepsilon))$ such that $(x(0, \varepsilon), y(0, \varepsilon))$ tends to $s_{+}$when $\varepsilon \rightarrow 0$.

Example (2.3) : Consider the differential system (1.4) with $a(t)=-\cos t, b(t)=\cos t, c(t)=\sin t \cos t$ and $h(t, x)=(x+1) \cos t$. These functions are $2 \pi$-periodic in the variable $t$. Then we obtain the functions $g(t, x)=$ 
$\int_{0}^{t} h(s, x) d s=(x+1) \sin t$ and $m(t)=\int_{0}^{t} a(s) d s=-\sin t$, which are $2 \pi$-periodic in the variable $t$. The polynomial system (3.16) of Theorem 1.2 for this differential system becomes

$$
2 y_{0}=0, \quad-4 x_{0}-2=0 .
$$

This system has the unique solution $s=(-1 / 2,0)$. Since the Jacobian (3.5) at this solution is 8 , the differential system (1.4) for $\varepsilon \neq 0$ sufficiently small has one periodic solution $(x(t, \varepsilon), y(t, \varepsilon))$ such that $(x(0, \varepsilon), y(0, \varepsilon))$ tends to $s$ when $\varepsilon \rightarrow 0$.

Example (2.4) : Consider the differential system (1.4) with $a(t)=\cos t, b(t)=2 \sin t, c(t)=\sin t+\cos t$ and $h(t, x)=x \sin t$. These functions are $2 \pi$-periodic in the variable $t$. Then we compute the functions $g(t, x)=$ $\int_{0}^{t} h(s, x) d s=x-x \cos t$ and $n(t)=\int_{0}^{t} b(s) d s=2-2 \cos t$, which are $2 \pi$-periodic in the variable $t$. For this differential system the polynomial system (3.17) of Theorem 1.2 is

$$
-4 x_{0}^{3}+2 x_{0}+2 y_{0}=0, \quad-36 x_{0}^{5}+22 x_{0}^{3}+12 x_{0}^{2} y_{0}-2 x_{0}-2 y_{0}=0 .
$$

This system has two non-zero real solutions $s_{ \pm}= \pm(1 / \sqrt{2}, 0)$. Since the Jacobian (3.5) is 12 for both solution, and system (1.4) for this example is invariant under the symmetry $(x, y) \rightarrow(-x,-y)$, it has for $\varepsilon \neq 0$ sufficiently small one periodic solution $(x(t, \varepsilon), y(t, \varepsilon))$ such that $(x(0, \varepsilon), y(0, \varepsilon))$ tends to $s_{+}$when $\varepsilon \rightarrow 0$.

Example (2.5) : Consider the differential system (1.4) with $a(t)=\sin t, b(t)=\cos t, c(t)=1+\cos t$ and $h(t, x)=\sin ^{2}(t+x)+\cos t$. These functions are $2 \pi$-periodic in the variable $t$. Then we compute the function $m(t)=\int_{0}^{t} a(s) d s=1-\cos t$, which is $2 \pi$-periodic in the variable $t$. For this differential system the polynomial system (3.18) of Theorem 1.2 becomes

$$
-2 x_{0}+2 y_{0}=0, \quad-2 x_{0}+1=0 .
$$

This system has the unique solution $s=(1 / 2,1 / 2)$. Since the Jacobian (3.5) for this solution is 4 , the differential system (1.4) for $\varepsilon \neq 0$ sufficiently small has one periodic solution $(x(t, \varepsilon), y(t, \varepsilon))$ such that $(x(0, \varepsilon), y(0, \varepsilon))$ tends to $s$ when $\varepsilon \rightarrow 0$.

Example (2.6) : Consider the differential system (1.4) with $a(t)=\sin t, b(t)=\cos t, c(t)=1+\cos t$ and $h(t, x)=\sin ^{2}(t+x)+\cos t$. These functions are $2 \pi$-periodic in the variable $t$. Then we obtain the function $n(t)=\int_{0}^{t} b(s) d s=\sin t$, which is $2 \pi$-periodic in the variable $t$. For this differential system the polynomial system (3.19) of Theorem 1.2 we obtain the system is

$$
2 y_{0}=0, \quad-3 x_{0}^{5}-2 y_{0}+1=0 .
$$

This system has the unique solution $s=\left(3^{4 / 5} / 3,0\right)$. Since the Jacobian (3.5) for this solution is $10 \pi^{2} 3^{1 / 5}$, the differential system (1.4) for $\varepsilon \neq 0$ sufficiently small has one periodic solution $(x(t, \varepsilon), y(t, \varepsilon))$ such that $(x(0, \varepsilon), y(0, \varepsilon))$ tends to $s$ when $\varepsilon \rightarrow 0$.

Example (2.7) : Consider the differential system (1.4) with $a(t)=2, b(t)=-\sin ^{2} t, c(t)=\cos t$ and $h(t, x)=$ $x \cos t$. These functions are $2 \pi$-periodic in the variable $t$. Then we compute the function $g(t, x)=\int_{0}^{t} h(s, x) d s=$ $x \sin t$, which is $2 \pi$-periodic in the variable $t . g_{x}(t, x)=\sin t$. For this differential system the polynomial system (3.20) of Theorem 1.2 becomes

$$
2 y_{0}=0, \quad x_{0}^{3}-5 x_{0}=0
$$


This system has two non-zero real solutions $s_{ \pm}= \pm(\sqrt{5}, 0)$. Since the Jacobian (3.5) is -20 for both solutions, and system (1.4) for this example is invariant under the symmetry $(x, y) \rightarrow(-x,-y)$ it has for $\varepsilon \neq 0$ sufficiently small one periodic solution $(x(t, \varepsilon), y(t, \varepsilon))$ such that $(x(0, \varepsilon), y(0, \varepsilon))$ tends to $s_{+}$when $\varepsilon \rightarrow 0$.

Example (2.8) : Consider the differential system (1.4) with $a(t)=-\cos ^{2} t, b(t)=2, c(t)=\cos t$ and $h(t, x)=$ $-x \cos t$. These functions are $2 \pi$-periodic in the variable $t$. For this differential system the polynomial system (3.21) of Theorem 1.2 is

$$
y_{0}=0, \quad-4 x_{0}^{3}+x_{0}=0 .
$$

It has two non-zero solutions $s_{ \pm}=(0, \pm 1 / 2)$. Since the Jacobian (3.5) is 2 for both solutions, and system (1.4) for this example is invariant under the symmetry $(x, y) \rightarrow(-x,-y)$ it has for $\varepsilon \neq 0$ sufficiently small one periodic solution $(x(t, \varepsilon), y(t, \varepsilon))$ such that $(x(0, \varepsilon), y(0, \varepsilon))$ tends to $s_{+}$when $\varepsilon \rightarrow 0$.

\section{Appendix 1: Averaging theory of first order}

In this appendix we recall one of the basic results from the averaging theory that we need for proving the results of this paper.

The averaging theory here presented provides an answer to the problem of the bifurcation of $T$-periodic solutions from differential systems of the form

$$
\mathbf{x}^{\prime}=F_{0}(t, \mathrm{x})+\varepsilon F_{1}(t, \mathrm{x})+\varepsilon^{2} F_{2}(t, \mathrm{x}, \varepsilon),
$$

with $\varepsilon=0$ to $\varepsilon \neq 0$ sufficiently small, where the $C^{2}$ functions $F_{0}, F_{1}: \mathbb{R} \times \Omega \rightarrow \mathbb{R}^{n}$ and $F_{2}: \mathbb{R} \times \Omega \times\left(-\varepsilon_{0}, \varepsilon_{0}\right) \rightarrow \mathbb{R}^{n}$ are $T$-periodic in the first variable, and the domain of definition of the differential system is $\Omega$ an open subset of $\mathbb{R}^{n}$. We assume that the unperturbed system

$$
\mathrm{x}^{\prime}=F_{0}(t, \mathrm{x})
$$

has a submanifold of dimension $n$ filled with periodic solutions.

Let $x(t, z, \varepsilon)$ be the solution of system (3.1) such that $x(0, z, \varepsilon)=0$. So, in particular $x(t, z, 0)$ is the solution of system (3.2) such that $x(0, z, 0)=0$.

The first variational equation of the unperturbed system (3.2) along the periodic solution $x(t, z, 0)$ is

$$
\mathrm{y}^{\prime}=D_{\mathrm{x}} F_{0}(t, \mathrm{x}(t, \mathrm{z}, 0)) \mathrm{y},
$$

where $\mathrm{y}$ is an $n \times n$ matrix. In what follows we denote by $M_{\mathrm{z}}(t)$ a fundamental matrix of the linear differential system (3.3).

We assume that there exists an open set $V$ with $\mathrm{C} \ell(V) \subset \Omega$ such that for each $z \in V$ the solution $x(t, z, 0)$ is $T$-periodic. So the set of periodic solutions contained in $V$ is isochronous for the system (3.1), i.e. it is a set formed only by periodic orbits having the same period $T$. Now an answer to the problem: which $T$-periodic solutions $x(t, z, 0)$ of system (3.2) contained in $V$ can be continued to T-periodic solutions $x(t, z, \varepsilon)$ of system (3.1) for $\varepsilon \neq 0$, is given in the following result.

Theorem 3.1. We assume that there exists an open and bounded set $V$ with $\mathrm{C} \ell(V) \subset \Omega$ such that for each $z \in V$ the solution $x(t, z, 0)$ is $T$-periodic, then we consider the average function $\mathcal{F}: V \longrightarrow \mathbb{R}^{n}$ defined by

$$
\mathcal{F}(z)=\int_{0}^{T} M_{z}^{-1}(t) F_{1}(t, x(t, z, 0)) d t .
$$

If there exists $\alpha \in V$ with $\mathcal{F}(\alpha)=0$ and

$$
\operatorname{det}((d \mathcal{F} / d z)(\alpha)) \neq 0
$$

then there exists a T-periodic solution $x(t, \varepsilon)$ of system (3.1) for $\varepsilon \neq 0$ sufficiently small such that when $\varepsilon \rightarrow 0$ we have that $x(0, \varepsilon) \rightarrow \alpha$.

Theorem 3.1 is due to Malkin and Roseau who proved it independently, see [11] and [15], respectively. For an easy proof of Theorem 3.1 see Corollary 1 of [3].

For more information about the averaging theory see the books of $[16,18]$. 


\section{Appendix 2}

We define in this appendix the polynomial systems in the variables $x_{0}$ and $y_{0}$ which appear in the statements of Theorem 1.1.

$$
\begin{aligned}
& -x_{0}^{3} \int_{0}^{T} n(t) e^{-k(t)} d t-x_{0} \int_{0}^{T} m(t) e^{-k(t)} d t+\int_{0}^{T} g\left(t, x_{0}\right) e^{-k(t)} d t+y_{0} \int_{0}^{T} e^{-k(t)} d t=0, \\
& -3 x_{0}^{5} \int_{0}^{T} n(t)^{2} e^{-k(t)} d t-x_{0}^{3} \int_{0}^{T} n(t)\left(4 m(t)-g_{x}\left(t, x_{0}\right)\right) e^{-k(t)} d t+3 x_{0}^{2} y_{0} \int_{0}^{T} n(t) . \\
& e^{-k(t)} d t+3 x_{0}^{2} \int_{0}^{T} n(t) g\left(t, x_{0}\right) e^{-k(t)} d t-x_{0} \int_{0}^{T} m(t)\left(m(t)-g_{x}\left(t, x_{0}\right)\right) e^{-k(t)} d t \\
& +\int_{0}^{T} g\left(t, x_{0}\right)\left(m(t)-g_{x}\left(t, x_{0}\right)\right) e^{-k(t)} d t+y_{0} \int_{0}^{T}\left(m(t)-g_{x}\left(t, x_{0}\right)\right) e^{-k(t)} d t=0 . \\
& -x_{0}^{3} \int_{0}^{T} n(t) e^{-k(t)} d t-x_{0} \int_{0}^{T} m(t) e^{-k(t)} d t+y_{0} \int_{0}^{T} e^{-k(t)} d t=0, \\
& -3 x_{0}^{5} \int_{0}^{T} n(t)^{2} e^{-k(t)} d t-4 x_{0}^{3} \int_{0}^{T} n(t) m(t) e^{-k(t)} d t+3 x_{0}^{2} y_{0} \int_{0}^{T} n(t) e^{-k(t)} d t \\
& -x_{0} \int_{0}^{T} m(t)^{2} e^{-k(t)} d t+\int_{0}^{T} h\left(t, x_{0}\right) e^{k(t)} d t+y_{0} \int_{0}^{T} m(t) e^{-k(t)} d t=0 . \\
& -x_{0} \int_{0}^{T} m(t) e^{-k(t)} d t+\int_{0}^{T} g\left(t, x_{0}\right) e^{-k(t)} d t+y_{0} \int_{0}^{T} e^{-k(t)} d t=0, \\
& -x_{0}^{3} \int_{0}^{T} b(t) e^{k(t)} d t-x_{0} \int_{0}^{T} m(t)\left(m(t)-g_{x}\left(t, x_{0}\right)\right) e^{-k(t)} d t+\int_{0}^{T} g\left(t, x_{0}\right)(m(t)- \\
& \left.g_{x}\left(t, x_{0}\right)\right) e^{-k(t)} d t+y_{0} \int_{0}^{T}\left(m(t)-g_{x}\left(t, x_{0}\right)\right) e^{-k(t)} d t=0 .
\end{aligned}
$$




$$
\begin{aligned}
& -x_{0}^{3} \int_{0}^{T} n(t) e^{-k(t)} d t+\int_{0}^{T} g\left(t, x_{0}\right) e^{-k(t)} d t+y_{0} \int_{0}^{T} e^{-k(t)} d t=0, \\
& -3 x_{0}^{5} \int_{0}^{T} n(t)^{2} e^{-k(t)} d t+x_{0}^{3} \int_{0}^{T} n(t) g_{x}\left(t, x_{0}\right) e^{-k(t)} d t+3 x_{0}^{2} y_{0} \int_{0}^{T} n(t) e^{-k(t)} d t+ \\
& 3 x_{0}^{2} \int_{0}^{T} n(t) g\left(t, x_{0}\right) e^{-k(t)} d t-x_{0} \int_{0}^{T} a(t) e^{k(t)} d t-\int_{0}^{T} g\left(t, x_{0}\right) g_{x}\left(t, x_{0}\right) e^{-k(t)} d t \\
& -y_{0} \int_{0}^{T} g_{x}\left(t, x_{0}\right) e^{-k(t)} d t=0 \\
& -x_{0} \int_{0}^{T} m(t) e^{-k(t)} d t+y_{0} \int_{0}^{T} e^{-k(t)} d t=0, \\
& -x_{0}^{3} \int_{0}^{T} b(t) e^{k(t)} d t-x_{0} \int_{0}^{T} m(t)^{2} e^{-k(t)} d t+\int_{0}^{T} h\left(t, x_{0}\right) e^{k(t)} d t+y_{0} \int_{0}^{T} m(t) e^{-k(t)} d t=0 . \\
& -x_{0}^{3} \int_{0}^{T} n(t) e^{-k(t)} d t+y_{0} \int_{0}^{T} e^{-k(t)} d t=0 \\
& -3 x_{0}^{5} \int_{0}^{T} n(t)^{2} e^{-k(t)} d t+3 x_{0}^{2} y_{0} \int_{0}^{T} n(t) e^{-k(t)} d t-x_{0} \int_{0}^{T} a(t) e^{k(t)} d t+\int_{0}^{T} h\left(t, x_{0}\right) e^{k(t)} d t=0 . \\
& \int_{0}^{T} g\left(t, x_{0}\right) e^{-k(t)} d t+y_{0} \int_{0}^{T} e^{-k(t)} d t=0, \\
& -x_{0}^{3} \int_{0}^{T} b(t) e^{k(t)} d t-x_{0} \int_{0}^{T} a(t) e^{k(t)} d t-\int_{0}^{T} g\left(t, x_{0}\right) g_{x}\left(t, x_{0}\right) e^{-k(t)} d t-y_{0} \int_{0}^{T} g_{x}\left(t, x_{0}\right) \mathbf{e}^{-\mathbf{k}(\mathbf{t})} \mathbf{d} \mathbf{t}=0 . \\
& -x_{0}^{3} \int_{0}^{T} b(t) e^{k(t)} d t-x_{0} \int_{0}^{T} a(t) e^{k(t)} d t+\int_{0}^{T} h\left(t, x_{0}\right) e^{k(t)} d t=0, \\
& y_{0}=0 \text {. }
\end{aligned}
$$




\section{Appendix 3}

We define in this appendix the polynomial systems in the variables $x_{0}$ and $y_{0}$ which appear in the statements of Theorem 1.2.

$$
\begin{aligned}
& -x_{0}^{3} \int_{0}^{T} n(t) d t-x_{0} \int_{0}^{T} m(t) d t+\int_{0}^{T} g\left(t, x_{0}\right) d t+T y_{0}=0, \\
& -3 x_{0}^{5} \int_{0}^{T} n(t)^{2} d t-x_{0}^{3} \int_{0}^{T} n(t)\left(4 m(t)-g_{x}\left(t, x_{0}\right)-c(t)\right) d t+3 x_{0}^{2} y_{0} \int_{0}^{T} n(t) d t+ \\
& 3 x_{0}^{2} \int_{0}^{T} n(t) g\left(t, x_{0}\right) d t-x_{0} \int_{0}^{T} m(t)\left(m(t)-g_{x}\left(t, x_{0}\right)-c(t)\right) d t+\int_{0}^{T} g\left(t, x_{0}\right)(m(t) \\
& \left.-g_{x}\left(t, x_{0}\right)-c(t)\right) d t+y_{0} \int_{0}^{T}\left(m(t)-g_{x}\left(t, x_{0}\right)-c(t)\right) d t=0 . \\
& -x_{0}^{3} \int_{0}^{T} n(t) d t-x_{0} \int_{0}^{T} m(t) d t+T y_{0}=0 \\
& -3 x_{0}^{5} \int_{0}^{T} n(t)^{2} d t-x_{0}^{3} \int_{0}^{T} n(t)(4 m(t)-c(t)) d t+3 x_{0}^{2} y_{0} \int_{0}^{T} n(t) d t-x_{0} \int_{0}^{T} m(t)(m(t)- \\
& c(t)) d t+\int_{0}^{T} h\left(t, x_{0}\right) d t+y_{0} \int_{0}^{T}(m(t)-c(t)) d t=0 \\
& -x_{0} \int_{0}^{T} m(t) d t+\int_{0}^{T} g\left(t, x_{0}\right) d t+T y_{0}=0 \\
& -x_{0}^{3} \int_{0}^{T} b(t) d t-x_{0} \int_{0}^{T} m(t)\left(m(t)-g_{x}\left(t, x_{0}\right)-c(t)\right) d t+\int_{0}^{T} g\left(t, x_{0}\right)\left(m(t)-g_{x}\left(t, x_{0}\right)-\right. \\
& c(t)) d t+y_{0} \int_{0}^{T}\left(m(t)-g_{x}\left(t, x_{0}\right)-c(t)\right) d t=0
\end{aligned}
$$




$$
\begin{aligned}
& -x_{0}^{3} \int_{0}^{T} n(t) d t+\int_{0}^{T} g\left(t, x_{0}\right) d t+T y_{0}=0 \\
& -3 x_{0}^{5} \int_{0}^{T} n(t)^{2} d t+x_{0}^{3} \int_{0}^{T} n(t)\left(g_{x}\left(t, x_{0}\right)+c(t)\right) d t+3 x_{0}^{2} y_{0} \int_{0}^{T} n(t) d t+3 x_{0}^{2} \int_{0}^{T} n(t) \\
& g\left(t, x_{0}\right) d t-x_{0} \int_{0}^{T} a(t) d t-\int_{0}^{T} g\left(t, x_{0}\right)\left(g_{x}\left(t, x_{0}\right)+c(t)\right) d t-y_{0} \int_{0}^{T}\left(g_{x}\left(t, x_{0}\right)+c(t)\right) d t=0 . \\
& -x_{0} \int_{0}^{T} m(t) d t+T y_{0}=0 \\
& -x_{0}^{3} \int_{0}^{T} b(t) d t-x_{0} \int_{0}^{T} m(t)(m(t)-c(t)) d t+\int_{0}^{T} h\left(t, x_{0}\right) d t+y_{0} \int_{0}^{T}(m(t)-c(t)) d t=0 . \\
& -x_{0}^{3} \int_{0}^{T} n(t) d t+T y_{0}=0 \\
& -3 x_{0}^{5} \int_{0}^{T} n(t)^{2} d t+x_{0}^{3} \int_{0}^{T} c(t) n(t) d t+3 x_{0}^{2} y_{0} \int_{0}^{T} n(t) d t-x_{0} \int_{0}^{T} a(t) d t+\int_{0}^{T} h\left(t, x_{0}\right) d t- \\
& y_{0} \int_{0}^{T} c(t) d t=0 \\
& \int_{0}^{T} g\left(t, x_{0}\right) d t+T y_{0}=0 \\
& -x_{0}^{3} \int_{0}^{T} b(t) d t-x_{0} \int_{0}^{T} a(t) d t-\int_{0}^{T} g\left(t, x_{0}\right)\left(g_{x}\left(t, x_{0}\right)+c(t)\right) d t-y_{0} \int_{0}^{T}\left(g_{x}\left(t, x_{0}\right)+c(t)\right) d t=0 . \\
& -x_{0}^{3} \int_{0}^{T} b(t) d t-x_{0} \int_{0}^{T} a(t) d t+\int_{0}^{T} h\left(t, x_{0}\right) d t=0, \\
& y_{0}=0 .
\end{aligned}
$$




\section{Acknowledgements}

The second author is partially supported by the Ministerio de Economía, Industria y Competitividad, Agencia Estatal de Investigación grant MTM2016-77278-P (FEDER), the Agència de Gestió d'Ajuts Universitaris i de Recerca grant 2017SGR1617, and the H2020 European Research Council grant MSCA-RISE-2017-777911.

\section{References}

[1] R. Benterki ANd J. Llibre, Periodic solutions of the Duffing differential equation revisited via the averaging theory, J. of Nonlinear Modeling and Analysis 1 (2019), 10-26.

[2] R. Benterki And J. Llibre, Periodic solutions of a class of Duffing differential equations, to appear in J. of Nonlinear Modeling and Analysis.

[3] A. Buica, J.P. Françoise AND J. Llibre, Periodic solutions of nonlinear periodic differential systems with a small parameter, Comm. Pure and Appl. Anal. 6 (2007), 103-111.

[4] H.B. Chen AND Y. LI, Stability and exact multiplicity of periodic solutions of Duffing equations with cubic nonlinearities, Proc. Amer. Math. Soc. 135 (2007), 3925-3932.

[5] H. Chen AND Y. LI, Rate of decay of stable periodic solutions of Duffing equations, J. Differential Equations 236 (2007), 493-503.

[6] H. Chen AND Y. LI, Bifurcation and stability of periodic solutions of Duffing equations, Nonlinearity 21 (2008), 2485-2503.

[7] A. C. Lazer and P. J. Mckenna, On the existence of stable periodic solutions of differential equations of Duffing type, Proc. Amer. Math. Soc. 110 (1990), 125-133.

[8] S. LiANG, Exact multiplicity and stability of periodic solutions for a Duffing equation, Mediterr. J. Math. 10 (2013), 189-199.

[9] J. Llibre And A. Makhlouf, Periodic solutions for periodic second-order differential equations with variable potentials, J Appl. Anal. 24 (2018), 127-137,

[10] J. Llibre AND L. Roberto, On the periodic solutions of a class of Duffing differential equations, Discrete Contin. Dyn. Syst. 33 (2013), 277-282.

[11] I.G. Malkin, Some Problems of the theory of nonlinear oscillations, Gosudarstv. Izdat. Tehn-Teor. Lit. Moscow, 1956 (in Russian).

[12] J. Mawhin, Seventy-five years of global analysis around the forcedpendulum equation, in Equadiff, Brno. Proceedings, Brno: Masaryk University 9 (1997), 115-145.

[13] F.I. NJoku AND P. OMARI, Stability properties of periodic solutions of a Duffing equation in the presence of lower and upper solutions, Appl. Math. Comput 135 (2003), 471-490.

[14] R. Ortega, Stability and index of periodic solutions of an equation of Duffing type, Boo. Uni. Mat. Ital B 3 (1989), 533-546.

[15] M. Roseau, Vibrations non linéaires et théorie de la stabilité, (French) Springer Tracts in Natural Philosophy, Vol. 8 Springer-Verlag, Berlin-New York, 1966.

[16] J.A. Sanders, F. Verhulst and J. Murdock, Averaging method in nonlinear dynamical systems, Appl. Math. Sci., Vol. 59, Springer, New York, 2007.

[17] P.J. Torres, Existence and stability of periodic solutions of a Duffing equation by using a new maximum principale, Mediterr. J. Math. 1 (2004), 479-486.

[18] F. Verhulst, Nonlinear Differential Equations and Dynamical Systems, Universitext, Springer, New York, 1996.

[19] F. WANG AND H. ZHU, Existence, uniqueness and stability of periodic solutions of a Duffing equation under periodic and anti-periodic eigenvalues conditions, Taiwanese J of Math. 19 (2015), 1457-1468. 